05,12

\title{
Особенности импульсного перемагничивания несферической наночастицы с кубической анизотропией
}

\author{
(C) А.М. Шутый, Д.И. Семенцов \\ Ульяновский государственный университет, \\ Ульяновск, Россия \\ E-mail: shuty@mail.ru \\ Поступила в Редакцию 9 февраля 2021 г. \\ В окончательной редакции 9 февраля 2021 г. \\ Принята к публикации 14 февраля 2021 г.
}

Для наночастицы эллипсоидальной формы с кубической магнитной анизотропией выявлены условия установления различных равновесных состояний после действия коротким гауссовым импульсом магнитного поля. Переход к каждому из этих состояний может быть реализован за счет изменения параметров импульса. Выявлено существенное влияние на процессы перемагничивания отклонения формы частицы от сферической.

Ключевые слова: импульсное перемагничивание, прецессионная динамика, кубическая анизотропия, эллипсоидальная магнитная наночастица, динамическая бистабильность.

DOI: 10.21883/FTT.2021.07.51039.022

\section{1. Введение}

В последние десятилетия достигнут значительный прогресс в понимании свойств и динамических процессов в дипольно-связанных структурах магнитных наночастиц. На изменении равновесных состояний магнитного момента (МM) под воздействием импульса магнитного поля основана возможность записи информации на дипольных решетках [1-8]. Дискретность дипольных решеточных структур приводит к существенным отличиям равновесных состояний и их динамического поведения от аналогичных свойств монодоменных макрообъектов [9-14], что связано не только с локальным упорядочением наночастиц, но также с симметрией и магнитной анизотропией отдельной частицы. В работах [15-17] показано, что для изолированной сферической наночастицы с одноосной магнитной анизотропией при изменении длительности или пикового значения импульса имеет место периодическая реализация условий перемагничивания (ПМ).

В настоящей работе на основе численного решения уравнений Ландау-Лифшица исследуется прецессионная динамика отклика ММ на импульсное воздействие эллипсоидальной монодоменной частицы с кубической магнитной анизотропией. В зависимости от знака константы анизотропии и формы частицы (вытянутой или сплюснутой) определяются условия установления после действия импульса возможных равновесных состояний ММ-частицы. Для сплюснутых частиц с отрицательной константой кубической анизотропии выявлена особенность зависимости от параметров импульса реализации перемагничивания/неперемагничивания (ПМ/НПМ).

\section{2. Энергия наночастицы}

Для монодоменной частицы эллипсоидальной формы, обладающей магнитной анизотропией, энергия ММ m запишется в виде суммы энергий во внешнем поле $\mathbf{H}$, анизотропии и полей рассеяния

$$
W(\mathbf{m})=-\mathbf{m H}+W_{a}(\mathbf{m})+W_{s}(\mathbf{m}) .
$$

Здесь энергия кубической анизотропии частицы имеет вид

$$
W_{a}(\mathbf{m})=-K_{1} \frac{V}{m^{4}}\left(m_{x}^{2} m_{y}^{2}+m_{x}^{2} m_{z}^{2}+m_{y}^{2} m_{z}^{2},\right)
$$

где $K_{1}-$ первая константа кубической анизотропии, $V$ - объем частицы. При $K_{1}>0$ оси легкого намагничивания ориентированы по кристаллографическим осям типа [100], при $K_{1}<0$ легкие оси ориентированы по осям [111]. Далее будем считать, что три оси типа [100] совпадают с осями координат.

Энергия полей рассеяния $W_{s}(\mathbf{m})=\mathbf{m} \hat{N} \mathbf{m} / 2 V$, где $\hat{N}-$ диагональный тензор размагничивающих коэффициентов. Для сферической частицы $N_{x}=N_{y}=N_{z}=4 \pi / 3$, для сплюснутого по оси $X$ эллипсоида

$$
\frac{\Delta N}{4 \pi}=\frac{3}{2\left(1-n^{2}\right)}\left\{1-\frac{n}{\sqrt{1-n^{2}}} \arcsin \sqrt{1-n^{2}}\right\}-\frac{1}{2},
$$

где $\Delta N=N_{x}-N_{\perp}>0, N_{\perp}=N_{y}=N_{z}, n=l_{x} / l_{\perp}<1$, $l_{x}$ и $l_{y}=l_{z}=l_{\perp}-$ размеры полуосей. Для вытянутого эллипсоида $n>1, \Delta N<0$ и

$$
\frac{\Delta N}{4 \pi}=\frac{3}{2\left(n^{2}-1\right)}\left\{\frac{n}{\sqrt{n^{2}-1}} \ln \left(n+\sqrt{n^{2}-1}\right)-1\right\}-\frac{1}{2} .
$$



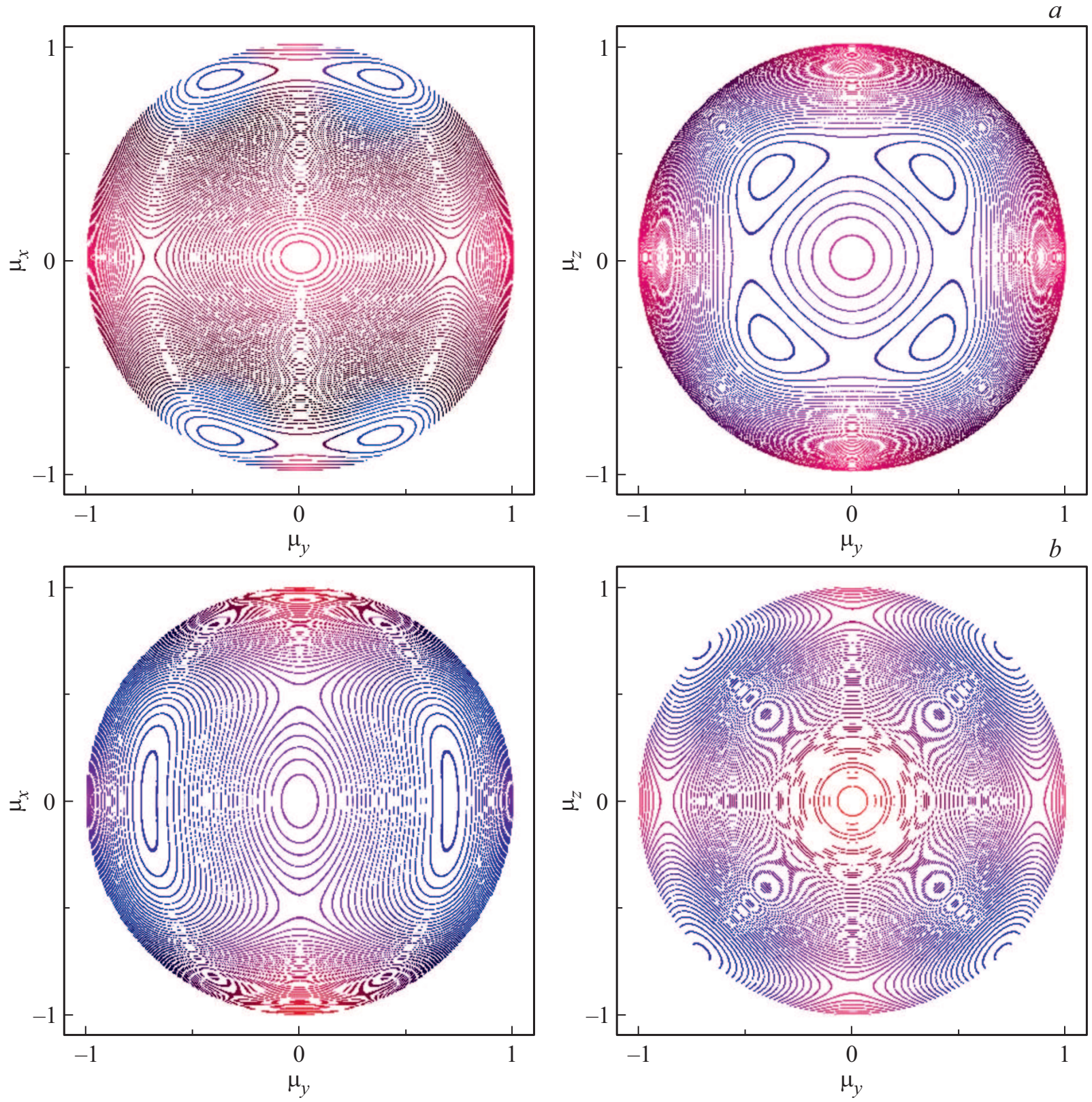

Рис. 1. Линий равной энергии ММ частицы с $n=0.95,1.05(a, b)$.

На рис. 1 на плоскостях $\left(\mu_{y}, \mu_{x}\right)$ и $\left(\mu_{y} \mu_{z}\right)$ приведены диаграммы линий равной энергии для ММ-частицы с параметром $n=0.95,1.05(a, b)$ в отсутствие внешнего магнитного поля при $K_{1}>0 \quad\left(k_{1}=2 K_{1} / J^{2}=1\right.$, $J=m / v)$. В случае сферической частицы шесть энергетических минимумов с $W_{\min }=-K_{1} V$ располагаются в полюсах диаграммы, а восемь максимумов с $W_{\min }=-K_{1} V / 3$ располагаются в точках выхода осей [111]. При $n<1$ энергетические максимумы смещаются к оси $X$. Минимумы, лежащие на оси $X$, уменьшаются по модулю, а лежащие в плоскости $Y Z$, возрастают. При $N>1$ ситуация противоположная, и восемь максимумов приближаются к плоскости $Y Z$, вырождаясь в четыре максимума для $n \geq 1.05$. В слу- чае $K_{1}<0$ минимумы и максимумы энергии на диаграммах меняются местами. При $k_{1}=-1$ диаграммы оказываются подобны приведенным с учетом смены положений минимумов и максимумов. При $n \leq 0.95$ восемь минимумов вырождаются в четыре, лежащих на плоскости $Y Z$.

\section{3. Динамика магнитного момента наночастицы}

Динамика ММ описывается уравнением

$$
\frac{\partial \mathbf{m}}{\partial t}=-\gamma \mathbf{m} \times \mathbf{H}^{\mathrm{eff}}+\frac{\alpha}{m} \mathbf{m} \times \frac{\partial \mathbf{m}}{\partial t},
$$



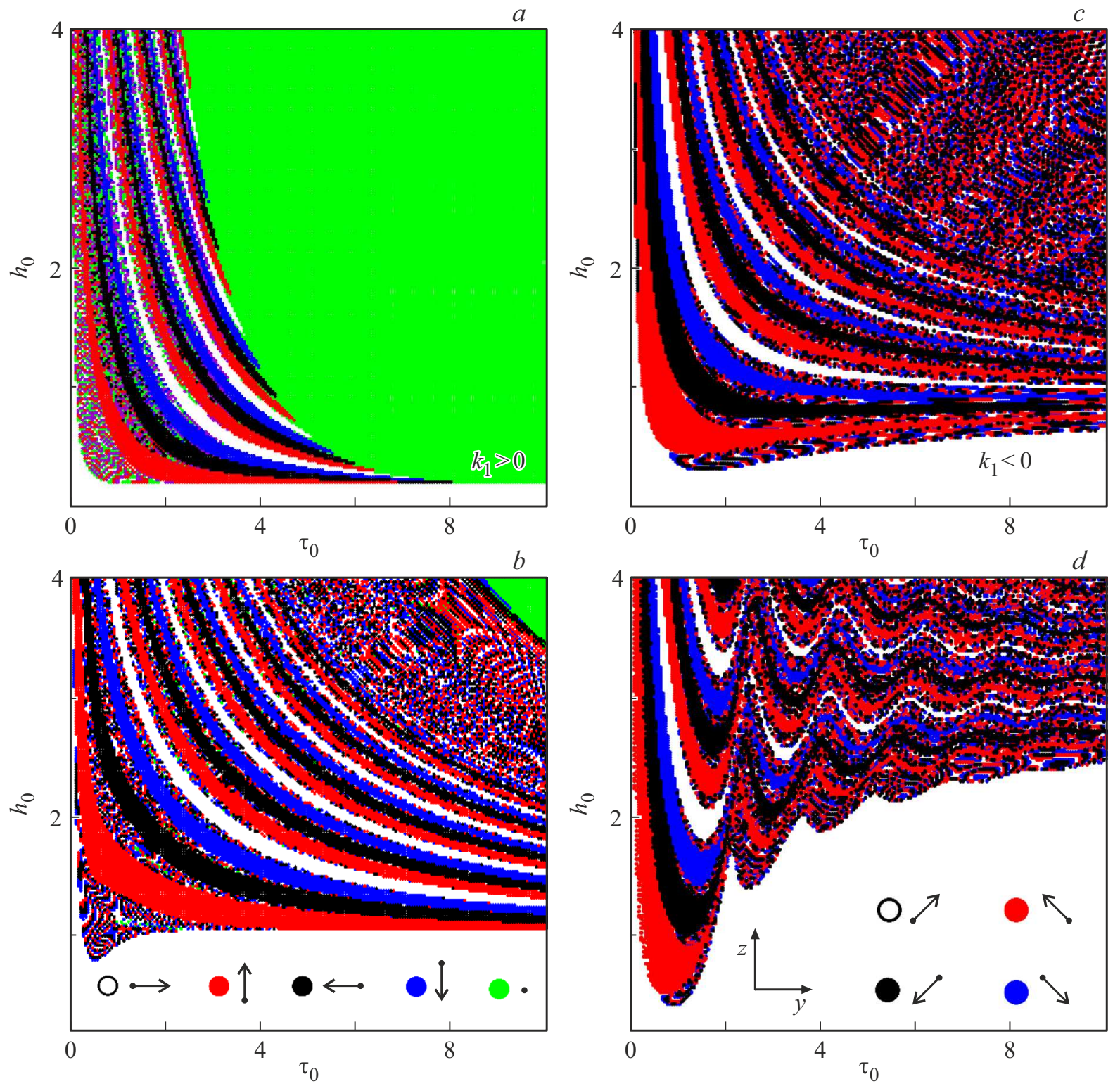

Рис. 2. Диаграммы зависимости от параметров импульса перемагничивания наночастицы с коэффициентом кубической анизотропии $k_{1}=1(a, b)$ и $k_{1}=-1(c, d)$ и параметром $n=1.05(a, c)$ и $n=0.95(b, d)$.

где $\gamma-$ гиромагнитное отношение, эффективное магнитное поле

$$
\mathbf{H}^{\mathrm{eff}}=-\partial W / \partial \mathbf{m}=\mathbf{H}+\mathbf{H}_{a}+\mathbf{m} \hat{N} / V .
$$

Рассмотрим поведение ММ-частицы при воздействии гауссова линейно поляризованного вдоль оси $X$ импульса магнитного поля

$$
h(\tau)=h_{0} \exp \left[-\left(\tau-\tau_{i}\right)^{2} / 2 \tau_{0}^{2}\right],
$$

где $h_{0}, \tau_{i}$ и $\tau_{0}-$ пиковое значение поля, временной сдвиг максимума и длительность импульса, $\tau_{i}=200$ (здесь введены безразмерные величины $h=H / J, \tau=\gamma J t$ ).

На рис. 2 для наночастиц с константой анизотропии $k_{1}=1(a, b)$ и $k_{1}=-1(c, d)$, а также фактором формы $n=1.05(a, c)$ и $n=0.95(b, d)$ приведены диаграммы, определяющие области параметров гауссова импульса $\left(h_{0}, \tau_{0}\right)$, при которых происходит (или не происходит) их перемагничивание. Поле импульса линейно поляризовано вдоль оси $X$. На диаграммах области определенного перемагничивания представляют собой чередующиеся полосы, между которыми располагаются области бистабильности. Внизу рисунка указано соответствие цвета областей конечной ориентации ММ.

В случае $k_{1}=1(a, b)$ в исходном состоянии ММ направлен вдоль оси $Y$. На диаграммах область параметров красной полосы (рисунки в цвете см. в эл. версии журнала) отвечает переходу ММ к ориентации вдоль направления $Z_{+}$, синей полосы - вдоль $Z_{-}$, черной полосы - вдоль направления $Y_{-}$, белая область отвечает 

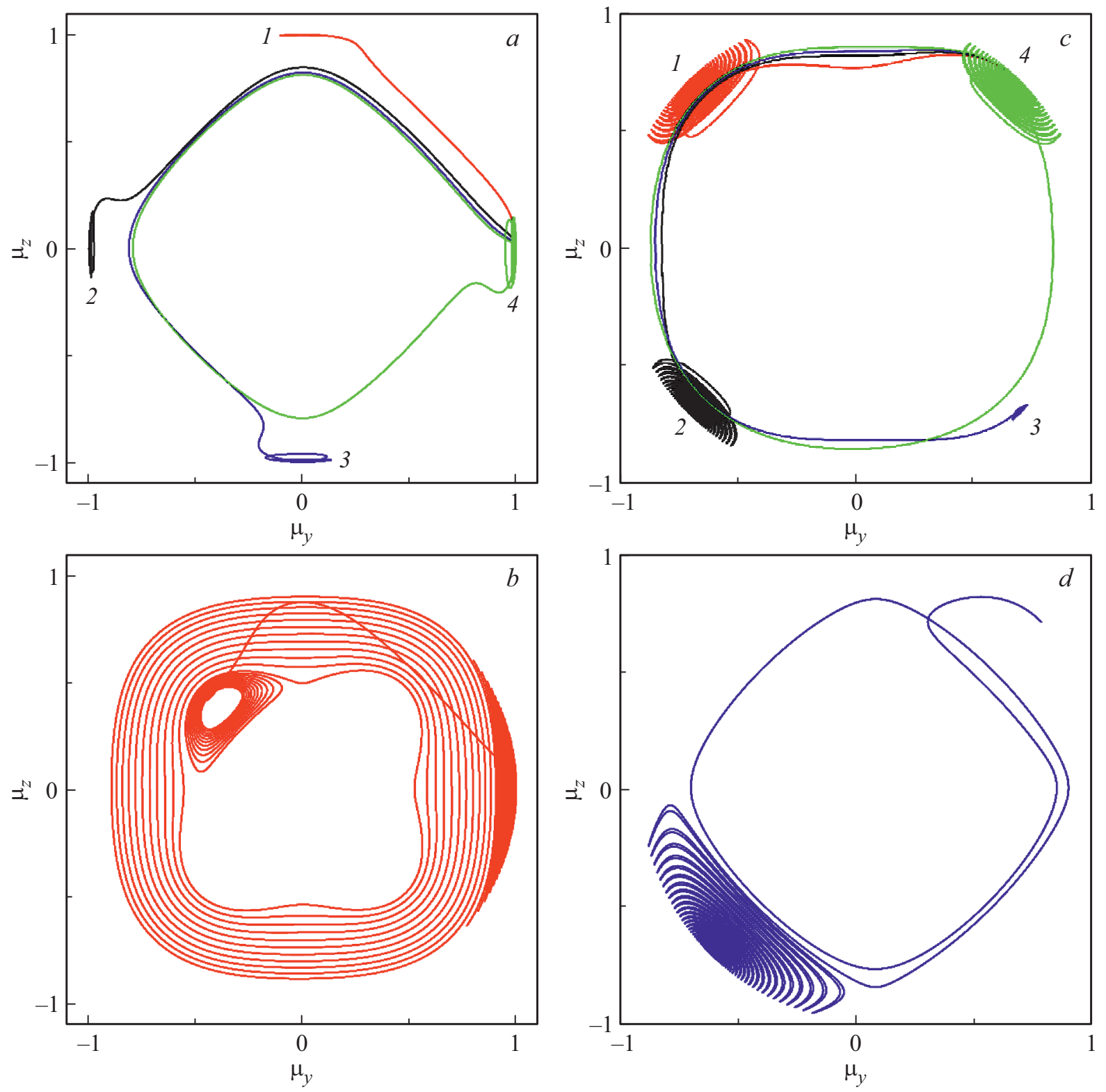

Рис. 3. Проекции траекторий магнитного момента кубически анизотропной наночастицы с $k_{1}=1, h_{0}=1.5, \tau_{-}=1,3,4.6,6(a$, кривые $1-4), \tau_{-}=2.2(b)$ и с $k_{1}=-1, \tau_{0}=1.5, h_{0}=0.9,1.2,1.5,2(c$, кривые $1-4)$ и $h_{0}=0.7(d) ; n=0.95$.

сохранению исходной ориентации вдоль направления $Y_{+}$. Полосы периодически повторяются, увеличиваясь в количестве с ростом значения параметров импульса. Над указанными полосами (в верхней правой зоне диаграмм) располагается область значений параметров (зеленая область), при которых ММ после действия импульса оказывается ориентированным вдоль оси $X$. Видно, что в случае вытянутого эллипсоида данная область значительно увеличивается. При этом ММ ориентируется по $X$ уже при достаточно малых параметрах импульса, так как $X$ является легкой осью для магнитного объекта такой формы. В случае же сплюснутого эллипсоида легкой является плоскость $Y Z$, поэтому при $n<1$ зеленая область диаграммы сильно сокращается.
На диаграммах также имеются области бистабильности (хаотически расположенных цветных точек). Для случая $n=1.05$ они располагаются при относительно малой длительности импульса вблизи границ областей перемагничивания, а в случае сплюснутого эллипсоида возникает обширная хаотическая область перед областью, отвечающей конечной ориентации ММ вдоль оси $X$. Данные области бистабильности отвечают параметрам, при которых под влиянием различных флуктуаций действие импульса может приводить к различным конечным ориентациям ММ. При увеличении значений параметров импульса полосы диаграмм, отвечающие перемагничиванию в определенном направлении, сужаются и исчезают, а области бистабильности расширя- 
ются и становятся преобладающими. Для реализации перемагничивания ММ к заданному направлению необходимо выбирать параметры импульса, относящиеся к соответствующим полосам.

В случае отрицательной константы анизотропии $(c, d)$ исходной ориентацией ММ является направление вдоль оси (111) с компонентами $\mu_{x, y, z} \approx 0.577$, а также соответствующие (по знаку компонент ММ) устойчивые равновесные ориентации для вытянутого и сплюснутого эллипсоидов. В результате действия импульса поля возможным оказывается перемагничивание к трем равновесным состояниям с положительной $x$-компонентой ММ и $\mu_{y} \approx 0.577, \mu_{z} \approx-0.577$ (отвечающие требуемым параметрам импульса точки диаграмм выделены красным цветом), $\mu_{y} \approx-0.577, \mu_{z} \approx-0.577$ (черный цвет), $\mu_{y} \approx-0.577, \mu_{z} \approx 0.577$ (синий цвет) и возвращение к исходной ориентации (белый цвет). В неприведенном здесь случае ориентации поля импульса в отрицательном направлении оси $X$ при исходной положительной $x$-компоненте ММ реализуется перемагничивание в четыре равновесных положения с изменением знака $x$-компоненты и в три положения с сохранением ее знака.

Важным отличием полученных диаграмм от обсуждавшихся в литературе ранее [18-20] являются два фактора. Во-первых, это значительное увеличение областей бистабильности, что обусловлено угловой близостью экстремальных точек на энергетических диаграммах (существенно превышающих соответствующие угловые расстояния для одноосных наночастиц). Во-вторых, это осцилляционный характер диаграмм в случае сплюснутого эллипсоида. Видно, что на диаграмме $(d)$ границы областей ПМ/НПМ определяются осцилляционными зависимостями $h_{0}\left(\tau_{0}\right)$. Это связано с сильно выраженным характером анизотропии типа „легкая плоскость“ за счет отрицательности константы анизотропии и сплюснутой формы наночастицы.

На рис. 3 приведены проекции траекторий ММ для нескольких случаев импульсного перемагничивания наночастиц с фактором формы $n=0.95$ и $k_{1}(a, b)$ и $k_{1}=-1(c, d)$. В случае положительной константы анизотропии на частицу воздействует импульс с параметрами $h_{0}=1.5, \tau_{0}=1,3,4.6,6$ (a, кривые $\left.1-4\right)$ и $\tau_{0}=2.2(b)$. В случаях $(a)$ ММ под действием импульса практически без прецессионной динамики за время, близкое к длительности импульса переходит от исходной ориентации вдоль положительного направления оси $Y$ к одному из трех равновесных положений или возвращается к исходному состоянию, в соответствии с полосой диаграммы перемагничивания, к которой относятся параметры импульса. Случай $(b)$ отвечает параметрам импульса из области бистабильности, в соответствии с чем после действия импульса ММ возвращается к исходному состоянию (после прецессии вокруг одной из областей с энергетическим максимумом и дальнейшей прецессии вблизи плоскости $Y Z$ ).
В случае отрицательной константы анизотропии различные конечные равновесные состояния ММ осуществляются в результате изменения амплитуды импульса $h_{0}=0.9,1.2,1.5,2$ (кривые 1-4) при одной величине его продолжительности $\tau_{0}=1.5(c)$. Продолжительность переходной прецессии при определенном перемагничивании увеличивается с приближением параметров импульса к границе соответствующей области диаграммы. В случае $(d)$ параметры импульса выбраны равными $h_{0}=0.7, \tau_{0}=1.5$ и относятся к области бистабильности. В результате ММ совершает один или несколько оборотов по большой траектории (в зависимости от длительности импульса) и после переходной прецессии с большой угловой амплитудой останавливается около одного из трех равновесных положений.

\section{4. Заключение}

Исследование импульсного воздействия на частицу с кубической магнитной анизотропией показало, что отклик и конечная ориентация ее ММ существенно зависят от параметров импульса магнитного поля. При изменении длительности или амплитуды перемагничивающего импульса имеет место чередование условий реализации одного из нескольких равновесных состояний ММ. Отклонение формы частицы от сферической на несколько процентов приводит к возникновению полей рассеяния, которые существенно изменяют как характеристические диаграммы перемагничивания, так и переходную динамику магнитного момента при его переходе под воздействием импульса к новому равновесному положению. Прецессионная переходная динамика при этом становится более сложной (состоящей из нескольких этапов) и в ряде случаев более продолжительной. Эллиптичность частицы (составляющая в рассматриваемых нами случаях 1-5\%) в несколько раз увеличивает области параметрических диаграмм, относящихся к состояниям динамической бистабильности, когда при одинаковом импульсном воздействии магнитный момент может перейти к различным равновесным положениям. Прецессионная динамика магнитного момента в условиях бистабильности характеризуется увеличенной продолжительностью и предельно большими амплитудами перемагничивания.

\section{Финансирование работы}

Работа поддержана Министерством науки и высшего образования РФ в рамках государственного задания № 0830-2020-0009.

\section{Конфликт интересов}

Авторы заявляют, что у них нет конфликта интересов. 


\section{Список литературы}

[1] R. Skomski. J. Phys.: Condens. Matter. 15, R841 (2003).

[2] E.Z. Meilikhov, R.M. Farzetdinova. JMMM 268, 237 (2004).

[3] В.А. Кособукин, Б.Б. Кричевцов. ФТТ 52, 759(2010).

[4] П.В. Бондаренко, А.Ю. Галкин, Б.А. Иванов. ЖЭТФ 139 , 1127 (2011).

[5] А.А. Фраерман. УФН 182, 1345 (2012).

[6] С.А. Дзян, Б.А. Иванов. ЖЭТФ 142, 969 (2012).

[7] S.A. Gudoshnikov, B.Ya. Liubimov, A.V. Popova, N.A. Usov. JMMM 324, 3690 (2012).

[8] M.F. Hansen, P.E. Jönsson, P. Nordblad, P. Svedlindh. J. Phys.: Condens. Matter. 14, 4901 (2012).

[9] T. Kiseleva, S. Zholudev, A. Novakova, T. Grigoryeva. Compos. Structures 138, 12 (2016).

[10] А.М. Шутый, Д.И. Семенцов. Письма в ЖЭТФ 99, 806 (2014).

[11] A.M. Shutyǐ, S.V. Eliseevay, D.I. Sementsov. Phys. Rev. B 91, 024421 (2015).

[12] A.M. Shuty̌̌, S.V. Eliseeva, D.I. Sementsov. JMMM 464, 76 (2018).

[13] A. Sukhov, J. Berakdar. Phys. Rev. B 79, 134433 (2009).

[14] Ю.И. Джежеря, К.О. Демишев, В.Н. Коренивский. ЖЭТФ 142, $2(8), 318$ (2012).

[15] H.W. Schumacher, C. Chappert, P. Crozat, R.C. Sousa, P.P. Freitas, J. Miltat, J. Fassbender, B. Hillebrands. Phys. Rev. Lett. 90, 1, 017201-4 (2003).

[16] А.М. Шутый, Д.И. Семенцов. Письма в ЖЭТФ 108, 11, 761 (2018)

[17] А.М. Шутый, Д.И. Семенцов. ЖЭТФ 156, 2 (8), 299 (2019).

Редактор Ю.Э. Китаев 